\title{
Effects of cytochrome P450 (CYP3A4 and CYP2C19) inhibition and induction on the exposure of selumetinib, a MEK1/2 inhibitor, in healthy subjects: results from two clinical trials
}

\author{
Angela W. Dymond ${ }^{1}$ - Karen So ${ }^{2} \cdot$ Paul Martin $^{1} \cdot$ Yifan Huang $^{3} \cdot$ Paul Severin $^{4}$. \\ David Mathews $^{5} \cdot$ Eleanor Lisbon $^{5} \cdot$ Gabriella Mariani $^{2}$
}

Received: 6 July 2016 / Accepted: 27 October 2016 / Published online: 26 November 2016

(C) The Author(s) 2016. This article is published with open access at Springerlink.com

\begin{abstract}
Purpose Two phase I, open-label trials in healthy subjects assessed whether co-administration with CYP3A4/CYP2C19 inhibitors, itraconazole/fluconazole (study A), or CYP3A4 inducer, rifampicin (study B), affects the exposure, safety/ tolerability and pharmacokinetics of selumetinib and its metabolite $N$-desmethyl selumetinib.

Methods In study A $(n=26)$, subjects received a single dose of selumetinib $25 \mathrm{mg}$ and, after 4 days of washout, were randomized to treatment 1 (itraconazole $200 \mathrm{mg}$ twice daily on days $1-11$ ) or treatment 2 (fluconazole $400 \mathrm{mg}$ on day 1 then $200 \mathrm{mg} /$ day on days 2-11) plus co-administration of singledose selumetinib $25 \mathrm{mg}$ on day 8 (selumetinib staggered $4 \mathrm{~h}$ after itraconazole/fluconazole dose); Twenty-one days after discharge/washout, subjects received the alternate treatment. In study B $(n=22)$, subjects received a single dose of
\end{abstract}

Angela Dymond, Paul Martin and David Mathews at the time of study conduct

Electronic supplementary material The online version of this article (doi:10.1007/s00228-016-2153-7) contains supplementary material, which is available to authorized users.

\section{Karen So}

karen.so@astrazeneca.com

Angela W. Dymond

Angela.Dymond@astrazeneca.com

1 AstraZeneca, Alderley Park, Macclesfield, Cheshire SK10 4TG, UK

2 AstraZeneca R\&D, AstraZeneca Global Medicines Development, Da Vinci Building, Melbourn, Royston, Hertfordshire SB8 6HB, UK

3 AstraZeneca, Gaithersburg, MD 20878, USA

4 Covance Laboratories, Inc., Madison, WI 53704, USA

5 Quintiles Phase I Services, Overland Park, KS 66211, USA selumetinib $75 \mathrm{mg}$ (day 1) then rifampicin $600 \mathrm{mg} /$ day (days 4-14) plus a single dose of selumetinib $75 \mathrm{mg}$ on day 12. Pharmacokinetic analysis and safety assessments were performed.

Results Selumetinib co-administered with itraconazole, fluconazole (selumetinib staggered $4 \mathrm{~h}$ after itraconazole/ fluconazole dose), or rifampicin was well tolerated. Selumetinib exposure was higher when co-administered with itraconazole or fluconazole (area under the plasma concentration-time curve (AUC) increased by 49 and 53\%, respectively; maximum plasma concentration $\left(C_{\max }\right)$ increased by 19 and $26 \%$, respectively) but lower when codosed with rifampicin (AUC and $C_{\max }$ decreased by 51 and $26 \%$, respectively) versus selumetinib dosed alone. Coadministration with itraconazole or rifampicin decreased $\mathrm{N}$ desmethyl selumetinib $\mathrm{AUC}_{(0-\mathrm{t})}$ (11 and 55\%, respectively), and $C_{\max }$ ( 25 and $18 \%$, respectively), with fluconazole, $\mathrm{AUC}_{(0-t)}$ increased by $40 \%$, but there was no effect on $C_{\max }$. Conclusions Co-administration of CYP3A4/CYP2C19 inhibitors will likely increase exposure to selumetinib, while CYP3A4 inducers will likely reduce its exposure.

Keywords Selumetinib · Exposure - Cytochrome P450 . Inhibition $\cdot$ Induction $\cdot$ Pharmacokinetic profile

\section{Introduction}

Selumetinib (AZD6244, ARRY-142886) is an oral, potent and selective, allosteric MEK1/2 inhibitor [1] in clinical development for a variety of different tumor types, including a phase III study in differentiated thyroid cancer [2] (NCT01843062) and a phase II registration study in neurofibromatosis type 1 [3] (NCT01362803). 
In vitro data from reaction phenotyping studies have shown that selumetinib can undergo metabolism by phase I (mainly oxidation through cytochrome P450 (CYP) enzymes) and phase II (conjugation with glucuronides) metabolic pathways (unpublished data ${ }^{1}$ ). CYP3A4 is the pre-dominant isoform responsible for selumetinib oxidative metabolism with CYP1A2, CYP2C9, CYP2C19, CYP2E1, and CYP3A5 that is also involved to a smaller extent. CYP3A4, in particular, is involved in the metabolism of a wide range of compounds, including various drugs and toxins [4-6]. Exposure of selumetinib may be influenced by CYP inhibitors or inducers, in particular by inhibitors or inducers of CYP3A4 or CYP2C19, potentially affecting the safety profile or efficacy of selumetinib. Potent CYP3A4 and CYP2C19 modulators include itraconazole and fluconazole (antifungal agents) and rifampicin (an antibiotic agent); these agents can be used as representative inhibitors or inducers of these CYP enzymes. Itraconazole and fluconazole are not totally selective towards CYP3A4 and CYP2C19, respectively, but demonstrate the most potent inhibition of these CYPs. Rifampicin induces many CYPs but exerts its most potent induction on CYP3A4. Since selumetinib will be given to patients who are potentially taking concurrent therapies, which could potentially modify the activity of these enzymes, assessing the exposure of selumetinib when taken together with such agents is extremely important for evaluating the effects on the safety profile and/or efficacy of selumetinib.

Here, we report two clinical trials in healthy subjects assessing the exposure of selumetinib and its metabolite: $\mathrm{N}$ desmethyl selumetinib when co-administered with the CYP3A4 or CYP2C19 inhibitors, itraconazole or fluconazole, respectively, or the CYP3A4 inducer, rifampicin.

\section{Subjects and methods}

Both phase I open-label, single-center trials in healthy subjects were performed by Quintiles Phase I Services (Overland Park, KS, USA). The trials were conducted in accordance with the ethical principles outlined in the Declaration of Helsinki and the International Conference on Harmonization Good Clinical Practice. Written informed consent was obtained from all subjects prior to any study-related procedures.

The primary study objectives were to investigate the effects of the potent CYP3A4 or CYP2C19 inhibitors, itraconazole $200 \mathrm{mg}$ and fluconazole $200 \mathrm{mg}$ ( $400 \mathrm{mg}$ on first day dose) at multiple doses, respectively, on the exposure of a single oral dose of selumetinib $25 \mathrm{mg}$ (NCT02093728), and the effects of CYP3A4 induction from multiple $600 \mathrm{mg}$ oral doses of rifampicin on the exposure of a single oral dose of selumetinib $75 \mathrm{mg}$ (NCT02046850). Secondary objectives included

\footnotetext{
${ }^{1}$ Report no. Be000021-21, data on file, AstraZeneca, Cheshire, UK
}

assessment of the pharmacokinetics (PKs) of $N$-desmethyl selumetinib following co-dosing of selumetinib with itraconazole, fluconazole, or rifampicin, and the safety and tolerability of selumetinib.

\section{Subjects}

For both studies, healthy male and female (of nonchildbearing potential) subjects aged 18-45 years, weighing $50-100 \mathrm{~kg}$, and with a body mass index of $18-30 \mathrm{~kg} / \mathrm{m}^{2}$ were eligible for enrollment. Additional information regarding the inclusion and exclusion criteria is included as Online Resource 1.

\section{Study design and experimental treatments}

A sample size of 20 evaluable healthy subjects was determined for each trial based on the desire to gain adequate information while exposing as few healthy subjects as possible to study procedures. As demonstrated in the expected $90 \%$ confidence intervals (CIs) for the geometric least squares mean ratios for area under the plasma concentration-time curve (AUC) and maximum plasma concentration $\left(C_{\max }\right)$, this sample size would provide an adequate precision for the estimation of potential drugdrug interaction effects. The expected $90 \%$ CIs were calculated using historical data. The within-subject estimates of the coefficient of variation $(\mathrm{CV})$ were derived from the relative bioavailability trial (NCT01635023) [7] of 16.9\% for AUC and 34.0\% for $C_{\max }$ for selumetinib (manuscript in preparation).

Twenty-six and 24 healthy subjects were planned for enrollment into the itraconazole/fluconazole (study A) and rifampicin (study B) studies, respectively, in order to ensure at least 20 subjects completed each study, assuming an approximate dropout rate of $20 \%$.

Selumetinib $75 \mathrm{mg}$ is the intended therapeutic dose and the maximum dose permitted in Western healthy subjects. A lower dose of selumetinib, $25 \mathrm{mg}$, was used in the itraconazole/ fluconazole trial to limit any increased exposure of selumetinib resulting from potential drug-drug interactions, to below the normal exposure at $75 \mathrm{mg}$. In both trials, the formulation of selumetinib used was identical to that in the ongoing phase III studies (oral capsules, containing $25 \mathrm{mg}$ freebase, equivalent of selumetinib Hyd-Sulfate; Patheon, Cincinnati, USA).

\section{Itraconazolelfluconazole trial (study A)}

The phase I, open-label, single-center, double-crossover-sequence, partially randomized (for assignment to treatment sequence), three-period itraconazole/fluconazole trial consisted of four visits to the study center (Fig. 1a). Subjects were screened during visit 1 , which is 28 days prior to visit 2 .

On day 1 of visit 2, subjects were randomized to one of two treatment sequences and admitted for residence at the study 
a

Itraconazole/fluconazole trial (Study A)

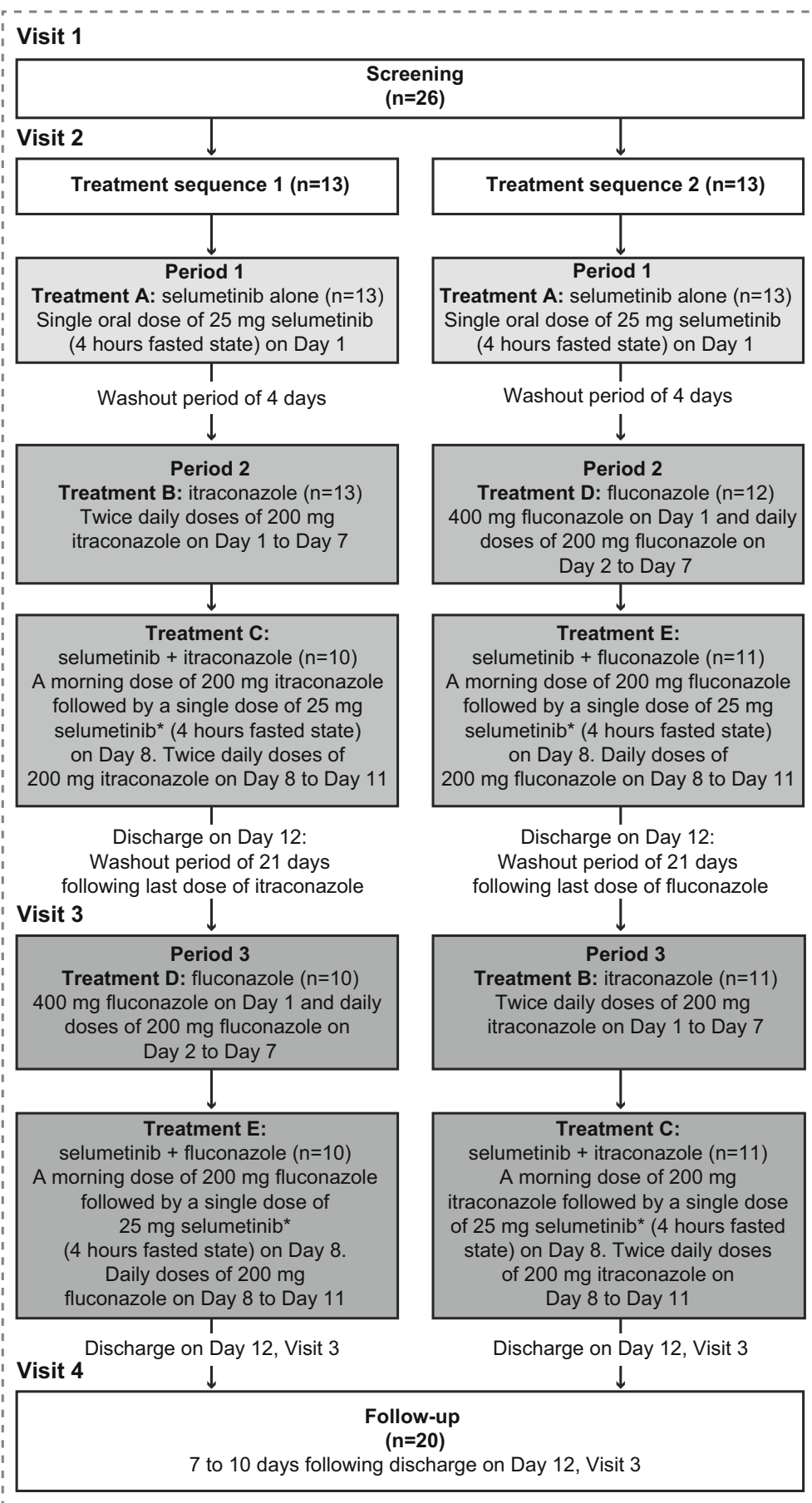

b

Rifampicin trial (Study B)

Visit 1

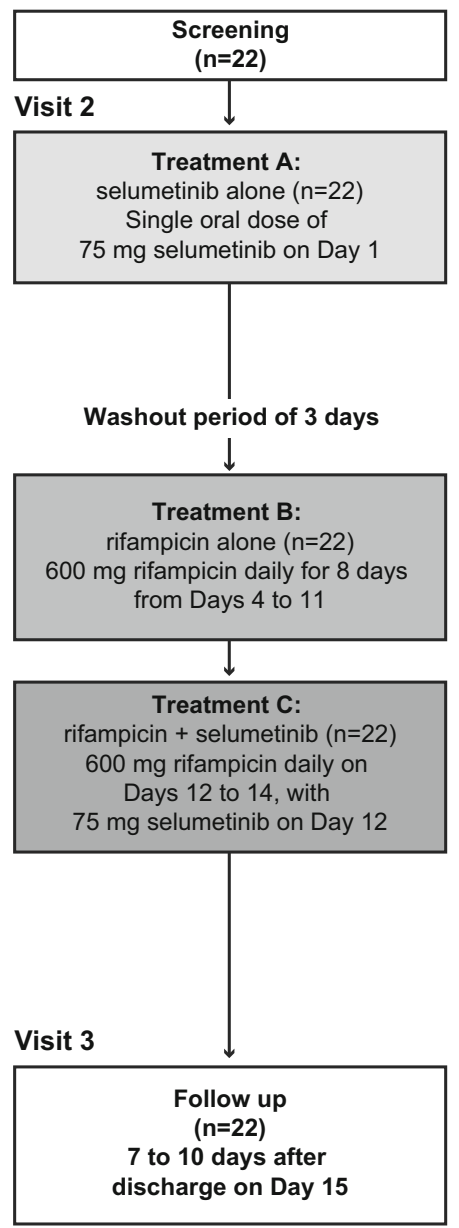

Fig. 1 Design of study A (itraconazole and fluconazole (a)) and study B (rifampicin (b)). Asterisk indicates that selumetinib staggered $4 \mathrm{~h}$ after itraconazole/fluconazole dose

center up to day 12; standardized meals were provided during residency. During dosing period 1, all subjects received a single dose of selumetinib $25 \mathrm{mg}$ followed by a 4 -day washout period. In dosing period 2 , during days $1-11$, subjects received either itraconazole $(200 \mathrm{mg}$ twice daily) or fluconazole (a $400 \mathrm{mg}$ loading dose on day 1 followed by $200 \mathrm{mg}$ once daily thereafter); all subjects received a second dose of selumetinib $25 \mathrm{mg}$ on day 8. Safety assessments were performed, and blood samples were collected for PK analysis up until 
day 12. Subjects were discharged on day 12 and completed a washout period of at least 21 days.

During visit 3 , dosing period 3 comprised days 1-11 when subjects received the alternate treatment regimen (fluconazole $400 \mathrm{mg}$ on day 1 followed by $200 \mathrm{mg}$ once daily thereafter or itraconazole $200 \mathrm{mg}$ twice daily) in a crossover fashion. All subjects received a second dose of selumetinib of $25 \mathrm{mg}$ on day 8. Subjects were discharged on day 12. A follow-up visit, visit 4 , was scheduled for 7-10 days after discharge from the study center.

Dose regimens for itraconazole and fluconazole were selected following consideration of the product Summary of Product Characteristics (SPCs) $[8,9]$ and internal expertise with the aim of maximizing the inhibitory effects on CYP3A4 and CYP2C19 while limiting exposure to the agents as appropriate. Subjects were administered with accepted clinical regimens of itraconazole (200 mg twice daily) or fluconazole (a $400 \mathrm{mg}$ loading dose and $200 \mathrm{mg}$ daily thereafter), respectively, for 7 days prior to selumetinib. Continued dosing of itraconazole or fluconazole for a further 3-4 days after selumetinib dosing ensured that the maximum enzyme inhibitory effects were maintained during the selumetinib sampling period. Selumetinib was administered $4 \mathrm{~h}$ after a light breakfast (approximately $4 \mathrm{~h}$ after the itraconazole or fluconazole dose), and subjects continued to fast at least $4 \mathrm{~h}$ post-dose. Fluids were not allowed from $1 \mathrm{~h}$ pre- until $1 \mathrm{~h}$ post-dose, with the exception of $240 \mathrm{~mL}$ water to swallow the capsules. Fluconazole was dosed with a light breakfast; there were no restrictions regarding dosing with food in the SPC [8]. Itraconazole was dosed with a light breakfast and dinner as per SPC guidelines to ensure complete absorption [9].

\section{Rifampicin trial (study B)}

The rifampicin open-label, fixed-sequence, single-center trial consisted of three visits to the study center (Fig. 1b). Subjects were screened during visit 1 , which is 28 days prior to visit 2 . During visit 2 , subjects received a single oral dose of selumetinib $75 \mathrm{mg}$ ( $3 \times 25 \mathrm{mg}$ capsules) on day 1 and resided at the study center up to day 15 . On day 4 , daily oral rifampicin $600 \mathrm{mg}$ (capsule) was commenced and continued up to day 14 , with a further single dose of selumetinib $75 \mathrm{mg}$ co-administered (at the same time) on day 12 . Safety assessments were performed, and blood samples were collected for PK analysis up until day 15 . Subjects were discharged from the study center on day 15 . The final visit, visit 3, was for follow-up and took place 7-10 days after discharge.

Subjects received daily dosing of rifampicin $600 \mathrm{mg}$ for 8 days to maximize the induction effect on CYP3A4 [10]. Selumetinib $75 \mathrm{mg}$ was administered in a fasted state; subjects were maintained in a fasted state overnight, for a minimum of $10 \mathrm{~h}$ until $4 \mathrm{~h}$ post-dose. Similarly, subjects were required to fast for at least $10 \mathrm{~h}$ prior to rifampicin administration and remained in the fasted state for a further $1 \mathrm{~h}$ post-dose. Fluids were not allowed from $1 \mathrm{~h}$ pre- until $1 \mathrm{~h}$ post-dose, with the exception of water needed to swallow investigational products.

\section{Pharmacokinetic assessments}

Serial blood samples $(2 \mathrm{~mL})$ to measure plasma selumetinib $\mathrm{PK}$ in the rifampicin trial were collected pre-selumetinib administration $(0 \mathrm{~h})$ and at $0.5,1.0,1.5,2.0,2.5,3.0,4.0,5.0$, $6.0,8.0,12.0,24.0,36.0,48.0$, and $72.0 \mathrm{~h}$ post-dose on days $1-4$ and $12-15$. The itraconazole/fluconazole trial included additional sampling time points, at 3.5 and $96.0 \mathrm{~h}$ postselumetinib administration on days $1-5$ of period 1 and days $8-12$ of periods 2 and 3 . Blood samples were collected prior to itraconazole or fluconazole administration on days 6 to 8 to determine trough itraconazole or fluconazole concentrations; rifampicin concentrations were measured at $2 \mathrm{~h}$ post-dose. In study B, the $4 \beta$-hydroxycholesterol to cholesterol concentration ratio was calculated as a biomarker of CYP3A4 induction. Blood samples $(4 \mathrm{~mL})$ to measure $4 \beta$ hydroxycholesterol and cholesterol prior to administration of rifampicin or selumetinib were collected on day 12 and before rifampicin administration on days 4 and 14. Samples were analyzed by Covance on behalf of AstraZeneca R\&D, using an appropriate bioanalytical method [e.g., 11, 12]. Additional information regarding the pharmacokinetic assessments, including bioanalytical methodology and assay performance, is included as Online Resource 2.

\section{Safety and tolerability}

Adverse events (reported by system organ class and preferred term using the Medical Dictionary for Regulatory Activities (MedDRA) version 17.0), vital signs, 12-lead electrocardiograms (ECGs), and clinical laboratory tests were recorded to assess safety and tolerability in both trials. Adverse events were collected from day 1 , period 1 of visit 2 , until the follow-up visit in each trial. Serious adverse events were collected from the time of informed consent until follow-up. Each adverse event was assigned to the period of study treatment in which it started or worsened. Additional information regarding safety and tolerability is included as Online Resource 3.

\section{Results}

\section{Trial populations}

Twenty-six and 22 healthy subjects were enrolled in the itraconazole/fluconazole and rifampicin studies, respectively (Online Resource 4 illustrates the passage of subjects through the studies). Subjects in both trials had an age range of 1844 years, and the majority were male $(>90 \%)$ and of similar mean body mass index $\left(26 \mathrm{~kg} / \mathrm{m}^{2}\right)$. Approximately half of all 
subjects in the itraconazole/fluconazole and rifampicin trials were White (50.0 and $45.5 \%$, respectively) and half were Black/African Americans (50 and 45.5\%, respectively); in the rifampicin trial, one subject $(4.5 \%)$ was classed as American Indian/Alaska Native and one subject (4.5\%) was classed as "Other."

In the itraconazole/fluconazole trial, all 26 subjects were randomized to one of two treatment sequences and all received study treatment. Twenty-one subjects $(80.8 \%)$ completed all treatment sequences, and $20(76.9 \%)$ completed the study (one subject was lost to follow-up). Five subjects discontinued partway through the study; three subjects (11.5\%) had protocol deviations (two subjects receiving selumetinib/itraconazole in period 2; one subject receiving selumetinib/fluconazole in period 2), one (3.8\%) violated the study site rules (the subject left and returned to the premises unescorted and unannounced during residential stay in period
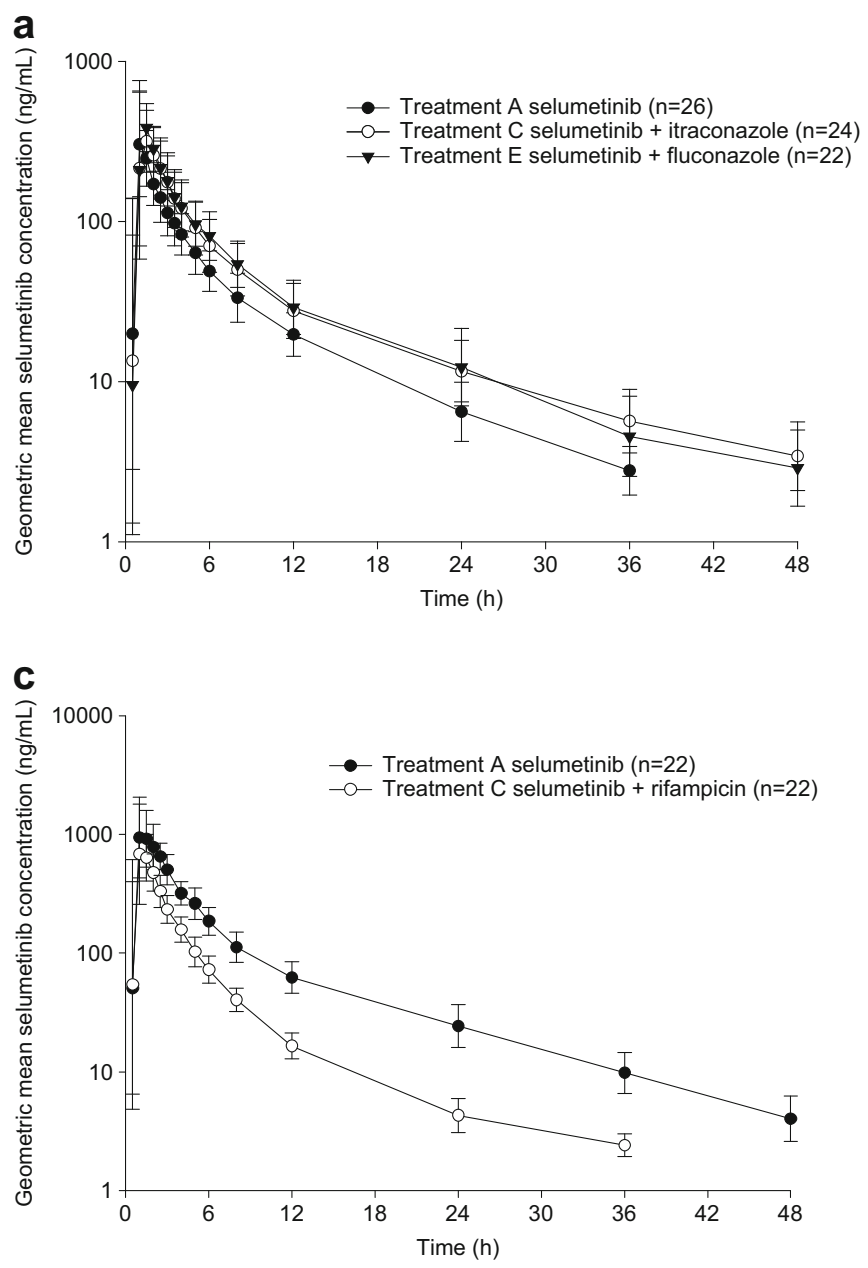

Fig. 2 Geometric mean plasma concentrations $(\mathrm{ng} / \mathrm{mL})$ versus time of selumetinib (a) and $\mathrm{N}$-desmethyl selumetinib (b) following single doses of selumetinib, and selumetinib co-dosed with itraconazole or fluconazole, and of selumetinib (c) and $N$-desmethyl selumetinib (d) following single doses of selumetinib, or selumetinib co-dosed with rifampicin. a, b Treatment A: $25 \mathrm{mg}$ selumetinib alone on day 1, treatment C: $200 \mathrm{mg}$ itraconazole twice daily on day 8 through day 11
2, fluconazole pre-dosing), and one withdrew consent (during period 2 selumetinib/itraconazole co-administration). In the rifampicin trial, all $22(100 \%)$ subjects received singlesequence treatment and completed the study.

\section{Pharmacokinetic results}

\section{Selumetinib co-dosed with itraconazole}

Trough plasma itraconazole concentration was well above CYP3A4 $\mathrm{IC}_{50}$ values [13], ranging from 595 to $776 \mathrm{ng} / \mathrm{mL}$ (geometric mean) on days 6 to 8 , indicating that sufficient exposure for inhibition of CYP3A4 was achieved. Selumetinib exposure increased when co-dosed with itraconazole (Fig. 2a; Table 1). AUC was increased by approximately $49 \%$ (90\% CI 40.4, 58.8), and $C_{\max }$ was increased by approximately $19 \%$ (90\% CI 4.2, 34.9) (Table 2). Co-dosing

b

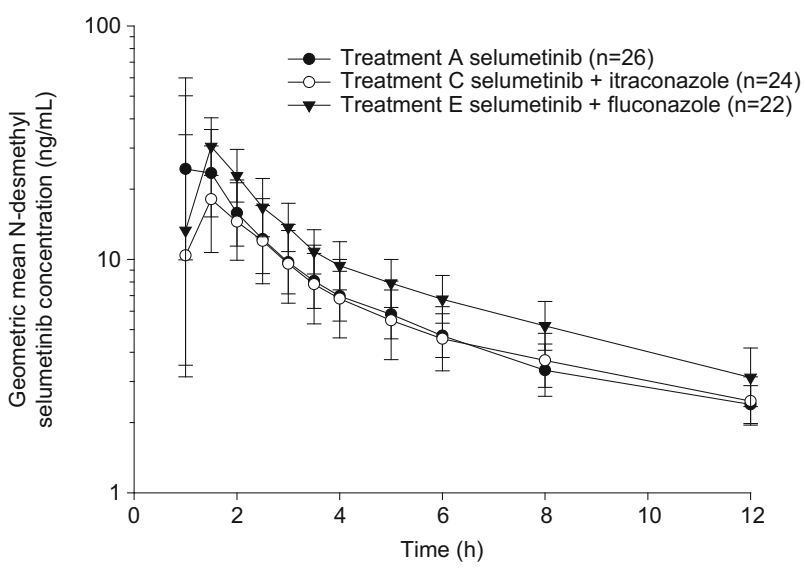

d

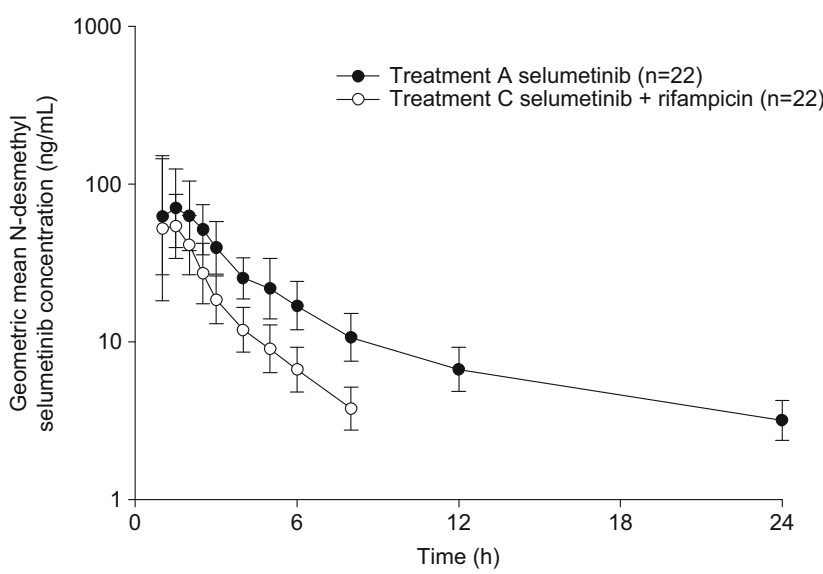

plus $25 \mathrm{mg}$ selumetinib on day 8 , treatment E: $200 \mathrm{mg}$ fluconazole once daily on day 8 through day 11 plus $25 \mathrm{mg}$ selumetinib on day 8 . c, d Treatment A: a single oral dose of $75 \mathrm{mg}(3 \times 25 \mathrm{mg})$ selumetinib administered under the fasted state on day 1 , treatment $\mathrm{C}$ : a single daily oral dose of $600 \mathrm{mg}$ rifampicin on days 12 to 14 , with $75 \mathrm{mg}$ selumetinib on day 12 under the fasted state 


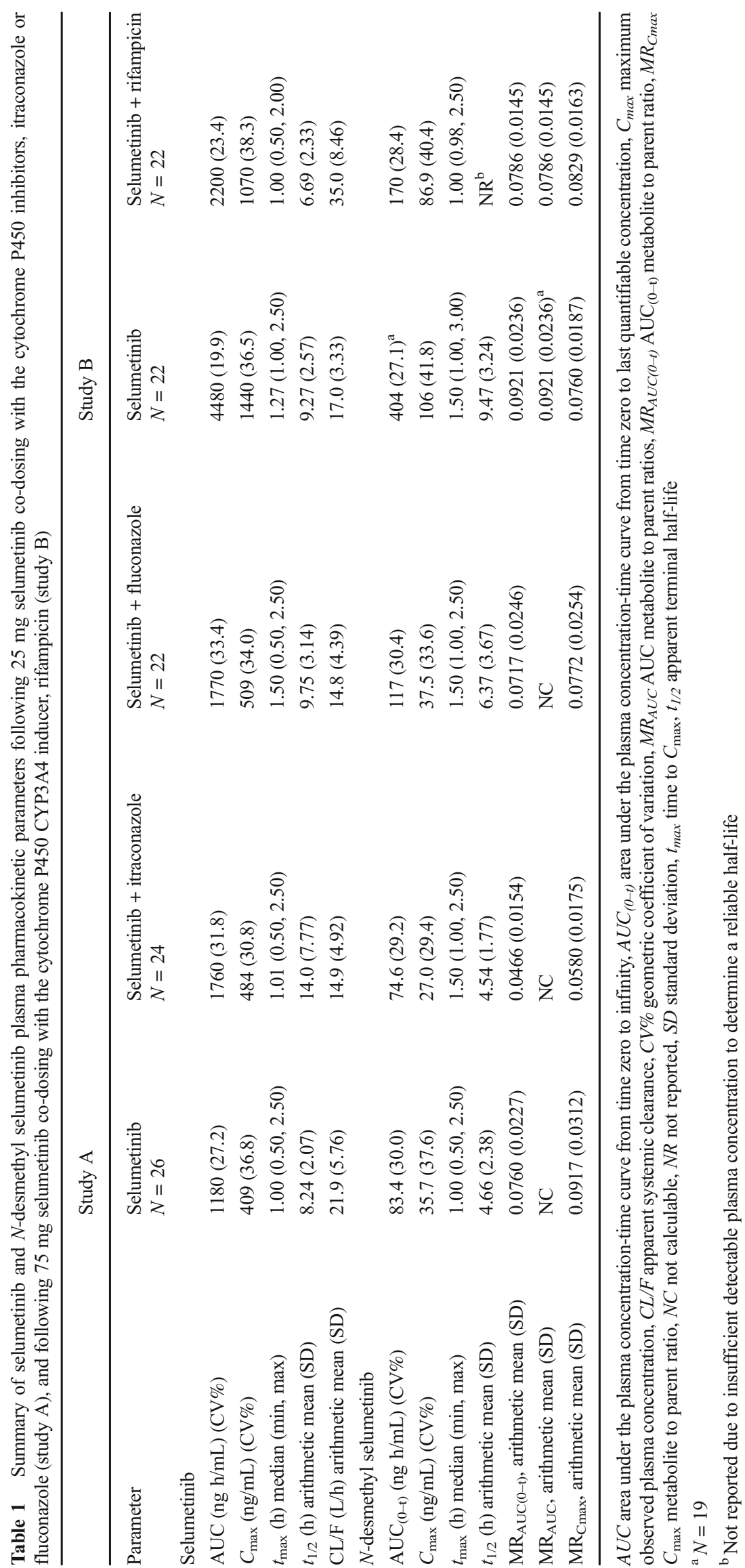


with itraconazole also prolonged selumetinib mean terminal half-life $\left(t_{1 / 2}\right)$ by $\sim 6 \mathrm{~h}$ and decreased apparent systemic clearance $(\mathrm{CL} / \mathrm{F})$ by approximately one third (Table 1$)$.

Itraconazole co-administration resulted in an initial decrease in $\mathrm{N}$-desmethyl selumetinib concentrations compared to selumetinib alone, but beyond the $2 \mathrm{~h}$ post-administration, concentrations were similar (Fig. 2b). Additionally, $N$ desmethyl selumetinib $\mathrm{AUC}_{(0-\mathrm{t})}$ and $C_{\max }$ were decreased with co-dosing of selumetinib and itraconazole compared with selumetinib alone by $11(90 \%$ CI $4.0,18.0)$ and $25 \%(90 \%$ CI $15.5,33.3$ ), respectively (Table 2). Itraconazole decreased the mean selumetinib metabolite to parent $C_{\max }$ and $\mathrm{AUC}_{(0-\mathrm{t})}$ ratios (Table 1).

Compared with administration of selumetinib alone, codosing with itraconazole did not influence median selumetinib time to $C_{\max }\left(t_{\max }\right)$; median difference in $N$-desmethyl selumetinib $t_{\max }$ was $0.25(0.00,0.50 \mathrm{~h} ; P=0.0366)$. This delay was considered not clinically relevant.

\section{Selumetinib co-dosed with fluconazole}

Trough plasma fluconazole concentration was generally within the CYP2C19 $\mathrm{IC}_{50}$ values [14], ranging from 5590 to $5730 \mathrm{ng} /$ $\mathrm{mL}$ (geometric mean) on days 6 to 8 , indicating that sufficient exposure for inhibition of CYP2C19 was achieved. Selumetinib exposure was increased when co-dosed with fluconazole compared with selumetinib alone (Fig. 2a; Table 1). AUC was increased by $53 \%(90 \% \mathrm{CI} 43.9,63.3)$, and $C_{\max }$ was increased by $26 \%$ (90\% CI 9.9, 43.4) (Table 2). Additionally, mean $t_{1 / 2}$ of selumetinib was slightly prolonged by $\sim 1.5 \mathrm{~h}$ and the mean CL/ $\mathrm{F}$ was decreased by approximately one third (Table 1).

Fluconazole co-administration resulted in an increase in $\mathrm{N}$ desmethyl selumetinib compared with selumetinib alone (Fig. 2b; Table 1). $\mathrm{AUC}_{(0-t)}$ was increased by $40 \%(90 \% \mathrm{CI}$ 29.1, 52.0), but there was no effect on $C_{\max }$ (Table 2). Only minor differences in the metabolite to parent ratios for $C_{\max }$ and AUC were detected (Table 1).

Compared with administration of selumetinib alone, codosing with fluconazole did not impact median selumetinib $t_{\max }$; median difference in $N$-desmethyl selumetinib $t_{\max }$ was $0.25 \mathrm{~h}(0.00,0.26 \mathrm{~h} ; P=0.0340)$. This difference was not considered clinically relevant.

\section{Selumetinib co-dosed with rifampicin}

Steady state rifampicin concentrations were achieved on the trial, as shown by levels similar to those reported in a previous study using similar doses of rifampicin [15] and the similar rifampicin concentrations across study days 7,9 , and 14 at $2 \mathrm{~h}$ post-dose (mean \pm standard deviation (SD) $8820 \pm 1870$, $7960 \pm 1660$, and $7900 \pm 1690 \mathrm{ng} / \mathrm{mL}$, respectively).

The $4 \beta$-hydroxycholesterol/cholesterol ratio (a biomarker of CYP3A4 induction) was increased on days 12 (3.4-fold) and 14 
(3.8-fold), following 9 and 11 days of continuous rifampicin administration, respectively, when compared to day 4 (pre-rifampicin). This increase indicated that rifampicin produced an adequate level of CYP3A4 induction to assess the effect on selumetinib exposure.

Selumetinib PK parameters following each treatment are summarized in Table 1, with statistical comparison of key PK exposure parameters summarized in Table 2. Selumetinib exposure was lower when co-dosed with rifampicin compared with selumetinib alone (Fig. 2c; Table 1). AUC was decreased by $51 \%(90 \% \mathrm{CI} 47.4,54.1)$, and $C_{\max }$ was reduced by $26 \%$ (90\% CI 16.7, 34.1) (Table 2).

$N$-desmethyl selumetinib exposure was lower when selumetinib was co-administered with rifampicin (Fig. 2d; Table 1), with a decrease of $55 \%(90 \%$ CI 50.9, 58.1) in $\mathrm{AUC}_{(0-\mathrm{t})}$ and $18 \%(90 \% \mathrm{CI} 6.4,28.8)$ in $C_{\text {max }}$ (Table 2). The decrease in $N$-desmethyl selumetinib plasma concentrations when co-administered with rifampicin compared with dosing alone was in proportion to the parent (Fig. 2c, d); hence, metabolite to parent mean ratios for AUC and $C_{\max }$ were not affected by co-dosing with rifampicin (Table 1).

Median selumetinib $t_{\max }$ was not affected by co-dosing with rifampicin (Table 1). Compared with selumetinib dosing alone, rifampicin co-administration resulted in an approximate $2.5 \mathrm{~h}$ shorter mean selumetinib $t_{1 / 2}$ $(9.27 \pm 2.57$ vs $6.69 \pm 2.33 \mathrm{~h})$ and a twofold increase in mean CL/F (Table 1).

$N$-desmethyl selumetinib $t_{\max }$ was slightly decreased when selumetinib was co-dosed with rifampicin (Table 1). $N$-desmethyl selumetinib concentrations were measurable to later time points when selumetinib was administered alone than when co-administered with rifampicin where concentrations were not quantifiable beyond $8 \mathrm{~h}$ post-dose. Hence, $N$-desmethyl selumetinib terminal $t_{1 / 2}$ estimation with rifampicin co-administration was less robust and is not presented here.

\section{Adverse effects}

No serious adverse events were reported during the itraconazole/fluconazole or the rifampicin PK studies in 48 subjects in total, and no subjects discontinued treatment due to adverse events.

In the itraconazole/fluconazole trial, $13 / 26$ subjects (50\%) reported at least one adverse event during the study. There were no major differences in incidence of adverse events reported in each treatment group. The highest number of adverse events was reported in subjects during treatment with selumetinib co-dosed with itraconazole ( $n=7 / 24 ; 29.2 \%)$, with headache ( $n=4 / 24 ; 16.7 \%$ ) being the most commonly reported adverse event in this treatment group. Five subjects $(5 / 24 ; 20.8 \%)$ reported adverse events during itraconazoleonly treatment, four each during fluconazole only (4/23;
$17.4 \%$ ) and selumetinib co-dosed with fluconazole treatment $(4 / 22 ; 18.2 \%)$, and two $(2 / 26 ; 7.7 \%)$ during selumetinib-only treatment. The majority of all reported adverse events were considered mild in severity by the investigators. One subject $(4.2 \%)$ reported a moderate adverse event, which is headache, during treatment with selumetinib co-dosed with itraconazole.

Overall, three subjects $(11.5 \%)$ reported adverse events considered related to selumetinib treatment by the investigator; diffuse alopecia and headache, both of mild severity, were each reported in one subject $(n=2 ; 8.3 \%)$ during selumetinib and itraconazole co-dosing, and acne of mild severity was reported in one $(4.5 \%)$ different subject during selumetinib co-dosed with fluconazole. Only one case of diffuse alopecia in the selumetinib co-dosed with itraconazole group and one case of vessel puncture site hemorrhage in the selumetinib codosed with fluconazole group, both of mild severity, were not resolved by the end of the study.

In the rifampicin trial, all 22 subjects $(100 \%)$ reported at least one adverse event; all adverse events were mild in severity and considered unrelated to selumetinib treatment. Adverse events were reported in all subjects during the period of rifampicin administration (days 4-11); chromaturia was reported by all of these subjects and resolved 2 to 3 days after rifampicin treatment was ceased. Chromaturia in subjects administered rifampicin was, as expected, in line with the SPC [16]. Four subjects (18.2\%) reported at least one adverse event during selumetinib-only treatment (arthralgia, $n=1$; diarrhea, $n=1$; increased alanine aminotransferase, $n=1$; pruritus, $n=1)$, and one subject $(4.5 \%)$ reported at least one adverse event (erythema, $n=1$ ) during selumetinib co-dosed with rifampicin. All of the adverse events were considered to be mild in intensity and resolved by the end of study completion.

No clinically significant or relevant trends or changes in clinical laboratory parameters, vital signs, ECGs, ophthalmological or physical assessments were detected in either trial.

\section{Discussion}

The drug-drug interaction (DDI) studies provide valuable knowledge into the potential treatment interactions during drug polypharmacy or concurrent medication use [17]. Such drug interactions may influence treatment safety and efficacy of the primary agent due to altered drug exposure and may provide important treatment considerations in the label.

In vitro data showed selumetinib to be mainly metabolized by CYP3A4 and CYP2C19; hence, it is important to understand whether the inhibition or induction of either of these enzymes influences selumetinib exposure in the clinic. With this in mind, a key objective for the phase I drug-drug interaction studies described was to assess the effect on the exposure of selumetinib following co-administration with either 
the CYP3A4 inhibitor itraconazole, the CYP2C19 inhibitor fluconazole, or the CYP3A4 inducer rifampicin, in healthy subjects. All of these agents have known potent enzyme modifying activities against these CYPs but can also modulate other CYPs. Results showed that selumetinib AUC increased by $\sim 50 \%$ when co-administered with either itraconazole (CYP3A4 inhibitor) or fluconazole (CYP2C19 inhibitor), and that selumetinib AUC was decreased by $\sim 50 \%$ by the CYP3A4 inducer rifampicin. These findings are in agreement with the in vitro data suggesting the involvement of these enzymes in the metabolism of selumetinib.

Inhibition of CYP3A4 by itraconazole or CYP2C19 by fluconazole affected levels of the metabolite, $N$-desmethyl selumetinib, differently. Co-administration of itraconazole resulted in a decrease in $N$-desmethyl selumetinib $C_{\max }(\sim 25 \%)$ and $\mathrm{AUC}_{0-\mathrm{t}}(\sim 10 \%)$, indicating that CYP3A4 is involved in the formation of $N$-desmethyl selumetinib. This was also reflected in a decrease in the ratio of metabolite to parent for peak concentration $\left(\mathrm{MR}_{\mathrm{Cmax}}\right)$ and overall exposure $\left(\mathrm{MR}_{\mathrm{AUC}(0-\mathrm{t})}\right)$ when selumetinib was co-administered with itraconazole than on its own.

Co-administration of fluconazole with selumetinib did not affect $N$-desmethyl selumetinib $C_{\text {max }}$, while $\mathrm{AUC}_{(0-\mathrm{t})}$ was increased, by about $40 \%$. With the inhibition of the CYP2C19 metabolic pathway by fluconazole, more $N$-desmethyl metabolite may have formed via an alternative metabolic route. This is also reflected in the generally unchanged mean metabolite to parent exposure ratios for $C_{\max }$ and $\mathrm{AUC}_{(0-\mathrm{t})}$ with fluconazole co-administration, suggesting minimal involvement of CYP2C19 in the formation of $N$-desmethyl selumetinib.

A similar decrease in $\mathrm{N}$-desmethyl selumetinib exposure to selumetinib exposure was observed when selumetinib was coadministered with rifampicin, indicating that CYP3A4 is also involved in $N$-desmethyl selumetinib metabolism. As rifampicin is also an inducer of other CYP450 enzymes to varying degrees [18, 19], including CYP2C19, the increase in selumetinib and $N$-desmethyl selumetinib metabolism may be due to a combination of both CYP3A4 and CYP2C19 inductions and potentially via other CYPs as well.

The dose of selumetinib was specifically selected to account for the potential changes in exposure on co-dosing with these potent CYP enzyme modifying agents; the resulting altered selumetinib AUC and $C_{\max }$ values did not exceed the safety exposure limit for healthy subjects that was derived from exposures in patients at the phase III selumetinib dose of $75 \mathrm{mg}$ twice daily. No safety concerns were identified between treatment groups.

The study design of the itraconazole and fluconazole trial proved efficient, using the same control arm for both treatments with no marked complications in recruitment or subject dropout. Moreover, there were no treatment discontinuations due to any adverse event. Observed adverse events were consistent with the safety profile of selumetinib and the additional study treatments. Selumetinib was well tolerated administered as either a single dose or when co-administered with itraconazole, fluconazole, or rifampicin. There were three adverse events (alopecia, headache, and acne, which were all of mild severity) considered related to selumetinib in the itraconazole/fluconazole trial and none in the rifampicin trial.

In the PK trials discussed, results using the CYP modulator probes for inhibition of CYP3A4 (itraconazole) or CYP2C19 (fluconazole), or induction of CYP3A4 (rifampicin), are informative of the potential exposure profile for selumetinib when co-administered with other CYP3A4/CYP2C19 inhibitors and CYP3A4 inducers in the clinical setting and, thus, are useful tool probes. By administering potent CYP3A4/CYP2C19 inhibitors and CYP3A4 inducers over a prolonged, multiple-dose regimen, this likely reflects a "worst case" situation for drugdrug interactions. The results presented here are encouraging and show that changes in the PK of selumetinib are relatively small $(\sim 50 \%)$. That said, the clinical significance of these potential changes will need to be considered together with safety and efficacy data from pivotal trials to determine any advice such as caution, additional monitoring, or dose adjustment that may be appropriate when co-administration is indicated.

A potential limitation of the current studies is that selumetinib was administered as a single dose ( 25 or $75 \mathrm{mg}$ ), alone, or in combination with one other treatment in healthy subjects. In the clinical setting, patients are likely to receive one or more treatments as multiple doses. However, despite this limitation, the data collected are sufficient to provide appropriate guidance in the drug label.

Another potential limitation was the separation of the itraconazole/fluconazole dosing versus selumetinib dosing to accommodate fed/fast advice for the respective treatments. Whilst it is not ideal to have this separation, it is not expected that the somewhat reduced itraconazole/fluconazole exposure at the time the selumetinib was dosed would diminish the inhibitory effects or the measured DDI too much, and it is believed that the data give a realistic assessment of the DDI risk.

\section{Conclusion}

Co-administration of the potent CYP3A4 inhibitor, itraconazole, or the potent CYP2C19 inhibitor, fluconazole, with selumetinib increased selumetinib exposure by $\sim 50 \%$. Thus, concomitant administration of potent CYP3A4 or CYP2C19 inhibitor with selumetinib may require caution. Co-administration of the potent CYP3A4 inducer, rifampicin, with selumetinib reduced exposure to selumetinib by $\sim 50 \%$. These study findings will be used to advise patient use in the clinical setting. 
Acknowledgements The trials were funded by AstraZeneca. The authors would like to acknowledge Jon Moran, $\mathrm{PhD}$, of iMed Comms, an Ashfield company, which is part of the UDG Healthcare plc, for the medical writing support that was funded by AstraZeneca.

Authors' contributions All authors fulfilled the ICMJE authorship criteria, including drafting, reviewing or revising the article, and approving the final version to be published. Additionally, the authors were involved as follows: $\mathrm{AD}$ and PM: study conception and design and data analysis and interpretation; EL and PS: data acquisition, analysis, and interpretation; GM and YH: data analysis and interpretation; DM: data acquisition; KS: study conception and design and data acquisition, analysis, and interpretation.

\section{Compliance with ethical standards}

Conflicts of interest $\mathrm{KS}, \mathrm{YH}$, and GM are employees of AstraZeneca and have stocks/options in AstraZeneca. AD and PM were employees of AstraZeneca at the time of study conduct and have stocks/options in AstraZeneca. EL, DM, and PS have no conflict of interest to declare.

Ethical approval All procedures performed in studies involving human participants were in accordance with the ethical standards of the institutional and/or national research committee and with the 1964 Helsinki Declaration and its later amendments or comparable ethical standards.

Informed consent Informed consent was obtained from all individual participants included in the study.

Open Access This article is distributed under the terms of the Creative Commons Attribution 4.0 International License (http:// creativecommons.org/licenses/by/4.0/), which permits unrestricted use, distribution, and reproduction in any medium, provided you give appropriate credit to the original author(s) and the source, provide a link to the Creative Commons license, and indicate if changes were made.

\section{References}

1. Yeh TC, Marsh V, Bernat BA et al (2007) Biological characterization of ARRY-142886 (AZD6244), a potent, highly selective mitogen-activated protein kinase kinase $1 / 2$ inhibitor. Clin Cancer Res 13(5):1576-1583

2. ClinicalTrials.gov (2015) Study comparing complete remission after treatment with selumetinib/placebo in patient with differentiated thyroid cancer (ASTRA) (NCT01843062). 2015. Available from: https://clinicaltrials.gov/ct2/show/NCT01843062. [11 Dec 2015]

3. Widemann, B.C., L.J. Marcus, M.J. Fisher et al., Phase I study of the MEK1/2 inhibitor selumetinib (AZD6244) hydrogen sulfate in children and young adults with neurofibromatosis type 1 (NF1) and inoperable plexiform neurofibromas (PNs). in J Clin Oncol. 2014. p. Abstract 10018 .

4. Miyamoto M, Yamashita T, Yasuhara Y et al (2015) Membrane anchor of cytochrome $\mathrm{P} 450$ reductase suppresses the uncoupling of cytochrome P450. Chem Pharm Bull (Tokyo) 63(4):286-294
5. Sevrioukova IF, Poulos TL (2015) Current approaches for investigating and predicting cytochrome P450 3A4-ligand interactions. Adv Exp Med Biol 851:83-105

6. Zanger UM, Schwab M (2013) Cytochrome P450 enzymes in drug metabolism: regulation of gene expression, enzyme activities, and impact of genetic variation. Pharmacol Ther 138(1):103-141

7. ClinicalTrials.gov (2015) A randomized, open label study to compare the current phase II and planned phase III capsule formulation of AZD6244 (AZD6244Formula). 2015. Available from: https:/clinicaltrials.gov/ct2/show/NCT01635023. [11 Dec 2015]

8. EMC (2015) Fluconazole Summary of Product Characteristics. 2015. Available from: http://www.medicines.org.uk/emc/medicine/25675. [20 May 2016]

9. EMC (2015) Itraconazole Summary of Product Characteristics. 2015 6/3/2015. Available from: http://www.medicines.org. uk/emc/medicine/28411. [2 Oct 2015]

10. Xu Y, Zhou Y, Hayashi M, Shou M, Skiles GL (2011) Simulation of clinical drug-drug interactions from hepatocyte CYP3A4 induction data and its potential utility in trial designs. Drug Metab Dispos 39(7):1139-1148

11. Leijen S, Soetekouw PM, Jeffry Evans TR et al (2011) A phase I, open-label, randomized crossover study to assess the effect of dosing of the MEK 1/2 inhibitor selumetinib (AZD6244; ARRY$142866)$ in the presence and absence of food in patients with advanced solid tumors. Cancer Chemother Pharmacol 68(6):16191628

12. Severin P, Bailey C, Chen M, Fisher A, Holmes V (2016) Determination of selumetinib, N-desmethyl selumetinib and selumetinib amide in human biological samples by LC-MS/MS. Bioanalysis 8(18):1919-1936

13. Templeton I, Peng C-C, Thummel KE, Davis C, Kunze KL, Isoherranen N (2010) Accurate prediction of dose-dependent CYP3A4 inhibition by itraconazole and its metabolites from in vitro inhibition data. Clin Pharmacol Ther 88(4):499-505

14. Dinger J, Meyer MR, Maurer HH (2014) Development of an in vitro cytochrome P450 cocktail inhibition assay for assessing the inhibition risk of drugs of abuse. Toxicol Lett 230:28-35

15. Ribera E, Azuaje C, Lopez RM et al (2007) Pharmacokinetic interaction between rifampicin and the once-daily combination of saquinavir and low-dose ritonavir in HIV-infected patients with tuberculosis. J Antimicrob Chemother 59(4):690-697

16. EMC (2014) Rifampicin Summary of Product Characteristics. 2014 15/9/2014. Available from: http://www.medicines.org. uk/emc/medicine/21223. [2 Oct 2015]

17. EMA. (2012) Guideline on the investigation of drug interactions 2012. 2012 1/01/2013. Available from: http://www.ema. europa.eu/docs/en GB/document library/Scientific guideline/2012/07/WC500129606.pdf. [2 Oct 2015]

18. Niemi M, Backman JT, Fromm MF, Neuvonen PJ, Kivisto KT (2003) Pharmacokinetic interactions with rifampicin : clinical relevance. Clin Pharmacokinet 42(9):819-850

19. Ridtitid W, Wongnawa M, Mahatthanatrakul W, Punyo J, Sunbhanich M (2002) Rifampin markedly decreases plasma concentrations of praziquantel in healthy volunteers. Clin Pharmacol Ther 72(5):505-513 